\title{
Direct imaging of a massive dust cloud around R Coronae Borealis ${ }^{\star}$
}

\author{
S. V. Jeffers ${ }^{1}$, M. Min ${ }^{1}$, L. B. F. M. Waters ${ }^{2,3}$, H. Canovas ${ }^{1}$, M. Rodenhuis ${ }^{1}$, M. de Juan Ovelar ${ }^{1}$, \\ A. L. Chies-Santos ${ }^{1}$, and C. U. Keller ${ }^{1}$ \\ 1 Sterrekundig Instituut Utrecht, Utrecht University, PO Box 80000, 3508 TA, Utrecht, The Netherlands \\ e-mail: S.V.Jeffers@uu.nl \\ 2 SRON, Netherlands Institute for Space Research, 3584 CA Utrecht, The Netherlands \\ 3 Astronomical Institute “Anton Pannekoek”, University of Amsterdam, PO Box 94249, 1090 GE Amsterdam, The Netherlands
}

Received 26 April 2011 / Accepted 16 November 2011

\section{ABSTRACT}

\begin{abstract}
We present recent polarimetric images of the highly variable star R CrB using ExPo and archival WFPC2 images from the HST. We observed $\mathrm{R} \mathrm{CrB}$ during its current dramatic minimum where it decreased more than 9 mag due to the formation of an obscuring dust cloud. Since the dust cloud is only in the line-of-sight, it mimics a coronograph allowing the imaging of the star's circumstellar environment. Our polarimetric observations surprisingly show another scattering dust cloud at approximately $1.3^{\prime \prime}$ or 2000 AU from the star. We find that to obtain a decrease in the stellar light of 9 mag and with $30 \%$ of the light being reemitted at infrared wavelengths (from R CrB's SED) the grains in R CrB's circumstellar environment must have a very low albedo of approximately $0.07 \%$. We show that the properties of the dust clouds formed around $\mathrm{R} \mathrm{CrB}$ are best fitted using a combination of two distinct populations of grains size. The first are the extremely small $5 \mathrm{~nm}$ grains, formed in the low density continuous wind, and the second population of large grains $(\sim 0.14 \mu \mathrm{m})$ which are found in the ejected dust clouds. The observed scattering cloud, not only contains such large grains, but is exceptionally massive compared to the average cloud.
\end{abstract}

Key words. circumstellar matter - supergiants - techniques: polarimetric - stars: individual: R Coronae Borealis

\section{Introduction}

$\mathrm{R}$ Coronae Borealis ( $\mathrm{R} \mathrm{CrB}$ ) stars are one of the most enigmatic classes of variable stars. They frequently show irregular declines in visual brightness of up to 9 mag due to the production of thick dust clouds. Such minima typically last for several months with the exception of R CrB's current minimum which has lasted for more than 3.5 years (since July 2007 - AAVSO). The photometric minima, caused by the obscuration of starlight by a dust cloud, are characterised by a rapid decline in brightness and a gradual return to normal brightness levels. Colour variations are also present with a reddening of the star during the decline, followed by the star becoming bluer during the phase of minimum brightness and then becoming redder during the rise to normal brightness levels. Variations in colour during a minimum have also been observed (e.g. Efimov 1988) and are attributed to pulsations, which are also are present in $\mathrm{R}$ CrB's light curve during maximum light.

The colour variations during obscuration can be attributed to the presence of a cool dust shell, which has been confirmed by observations of a strong IR-excess (Kilkenny \& Whittet 1984; Walker 1986). The cool dust shell has a temperature of $\approx 500-1000 \mathrm{~K}$ and $L$-band infrared photometric observations show that it still emits in the infrared even when the line-ofsight is obscured by a dust cloud (Feast 1986; Yudin et al. 2002).

* Based on observations made with the William Herschel Telescope operated on the island of La Palma by the Isaac Newton Group in the Spanish Observatorio del Roque de los Muchachos of the Instituto de Astrofisica de Canarias.
Feast (1979) notes that the $L$-band observations mirror the pulsation frequencies of the photosphere. The cool dust shell is assumed to be continuously replenished by randomly emitted dust clouds. The extinction of the ejected dust is different to that of the ISM, with an UV absorption peak at $2500 \AA$ as opposed to $2175 \AA$ A. Laboratory measurements and theoretical models show that this is due to small glassy or amorphous carbon grains (i.e. soot) formed in a hydrogen-poor environment (Hecht et al. 1984).

The formation of dust clouds on the R CrB star RY Sgr has been observed by de Laverny \& Mékarnia (2004) with NACO at the VLT. These observations show that the dust clouds are bright, contributing up to $2 \%$ of the stellar flux in the infrared ( $L$-band $4.05 \mu \mathrm{m})$, and are located in any direction at several hundred stellar radii from RY Sgr. de Laverny \& Mékarnia (2004) also note that the dust clouds might be very dense and optically thick close to the star. Leão et al. (2007) used mid-infrared interferometric observation of RY Sgr, with VLTI/MIDI, to show that the central star is surrounded by a circumstellar envelope with one bright dust cloud at 100 stellar radii (30 AU). This is notably the closest observed dust cloud around an R CrB star. More recently Bright et al. (2011) also used VLTI/MIDI to probe the circumstellar environments of RY Sgy, V CrA and V854 Cen at very small spatial scales (50 mas/400 $R_{\star}$ ). They find that their observations are consistent with a scenario of random dust ejection around the star, which over time creates a halo of dust.

The dust formation mechanism in these stars is not well understood. However, Crause et al. (2007) show that there is a correlation between the onset of brightness declines and $\mathrm{R} \mathrm{CrB}$ 's 
pulsation period, linking the expulsion of dust clouds and mass loss to internal stellar processes. This has also been shown to occur on other R CrB-type stars such as V854 Cen (Lawson et al. 1992) and RY Sgr (Pugach 1977). Observations show broad emission lines (Na D lines, Ca I H \& K and $388.8 \mathrm{~nm}$ He line), as the star's brightness decreases, indicating gas velocities of $\approx 200 \mathrm{~km} \mathrm{~s}^{-1}$. This is in agreement with observations of the He I $\lambda 10830$ line, which indicate a wind with a velocity of 200-240 $\mathrm{km} \mathrm{s}^{-1}$ (Clayton et al. 2003). They also note that the $\mathrm{P}$ Cygni profile is present during both maximum light and brightness decline. For $\mathrm{R} \mathrm{CrB}$, the dust grains are assumed to be formed away from the star, at approximately $20 R_{*}$, because at the stellar surface the temperature greatly exceeds the grain condensation temperature for carbon (Feast 1986; Fadeyev 1988). The dust clouds are then driven away from the star by radiation pressure. An alternative model proposes that the dust is formed close to the star and moves quickly away due to radiation pressure (originally proposed by Payne-Gaposchkin 1963). However, the conditions close to the star are far from the thermodynamic equilibrium necessary to form dust grains, though as discussed by Clayton (1996) and Donn (1967), the condensation temperature of carbon in a hydrogen deficient environment, such as on an R CrB star, is much higher than when hydrogen is present.

To further understand the circumstellar environment of $\mathrm{R} \mathrm{CrB}$ we have used imaging polarimetry observations with ExPo, the EXtreme POlarimeter (Rodenhuis, in prep.), to detect scattered starlight during its current minimum. This method is advantageous as starlight becomes polarised when it is scattered from circumstellar dust and particularly during obscuration can enable a detection of close-in circumstellar dust. In this paper we combine images of the dust cloud in scattered light, using imaging polarimetry, with archival images taken by the Hubble Space Telescope (HST) to determine the properties of the grains in the obscuring and scattering dust clouds.

In this paper we first describe the observations of $\mathrm{R} \mathrm{CrB}$ using ExPo and the HST in Sects. 2 and 3. In Sect. 4, we summarise the observational facts that we derive from the observations, which we use in Sect. 5 to model the properties of the dust grains. Our results are discussed in Sect. 6.

\section{Polarimetric imaging with ExPo}

\subsection{ExPo: instrumental setup}

The design of ExPo is based on the principle that light reflected by circumstellar material becomes linearly polarised and can be easily separated from the unpolarised light that originates from the central star. One of the design concepts of ExPo is the combination of fast modulation of polarisation states with dual-beam imaging. This setup significantly reduces the impact of flat field and seeing effects on the polarimetric sensitivity.

ExPo is a regular visitor instrument at the $4.2 \mathrm{~m}$ William Herschel Telescope. It has been designed and built at Utrecht University and has a $20^{\prime \prime} \times 20^{\prime \prime}$ field-of-view and a wavelength range of $500 \mathrm{~nm}$ to $900 \mathrm{~nm}$. The fast modulation of polarisation states is achieved with the combination of a ferroelectric liquid crystal (FLC), a cube beamsplitter and a EM-CCD camera. The FLC modulates the incoming light into two polarisation states separated by $90^{\circ}$, "A" and "B" states. The beam splitter separates each of these frames into two beams which are imaged on the EM-CCD as $A_{\text {left }}$ and $A_{\text {right }}$ followed by $B_{\text {left }}$ and $B_{\text {right }}$.
Table 1. Journal summarising ExPo and HST observations.

\begin{tabular}{lcccc}
\hline \hline Instrument & Date & Filter & Exp time(s) & No exp \\
\hline ExPo & 27 May 2010 & none & 0.028 & $4 \times 1000$ \\
HST/WFPC2 & 14 April 2009 & $F 55 W W \mathrm{~nm}$ & 7 & 1 \\
& & $F 555 W \mathrm{~nm}$ & 120 & 4 \\
& & $F 814 W \mathrm{~nm}$ & 16 & 1 \\
& & $F 814 W \mathrm{~nm}$ & 160 & 3 \\
\hline
\end{tabular}

\subsection{ExPo: observations and data analysis}

Observations of $\mathrm{R} \mathrm{CrB}$ were secured at the $4.2 \mathrm{~m}$ William Herschel Telescope on La Palma, as part of a larger observing run, from 22 to 27 May 2010. To obtain an image we take typically 4000 frames with an exposure time of $0.028 \mathrm{~s}$ per FLC angle i.e. $0^{\circ}, 22.5^{\circ}, 45^{\circ}$ and $67.5^{\circ}$. These observations are summarised in Table 1. A set of flat fields is taken at the beginning and end of each night and a set of darks is taken at the beginning of each observation.

All of the images are dark subtracted, and corrected for flat fielding, bias and cosmic ray effects. Once the polarization images are free of instrumental effects, they are carefully aligned and averaged. Polarised images are obtained after applying a double-difference approach to the two beams and alternating " $\mathrm{A}$ " and "B" frames, i.e.

$P=0.5\left(P_{\mathrm{A}}-P_{\mathrm{B}}\right)=0.5\left(\left(A_{\mathrm{L}}-A_{\mathrm{R}}\right)-\left(B_{\mathrm{L}}-B_{\mathrm{R}}\right)\right)$.

Special care was taken in aligning the $\mathrm{R} \mathrm{CrB}$ images, since its polarized image shows one clear dust cloud rather than an extended polarized source such as a disk or a shell. To minimise guiding effects, the images were first aligned with a template. Secondly, to minimize intensity gradient effects, the images were aligned with an accuracy of a third of a pixel. This results in the cloud structure showing more detail than using other techniques such as aligning the images with respect to the brightest speckle. Finally, the reduced images are calibrated using the method of (Rodenhuis et al., in prep.) to produce Stokes $Q$ and $U$ images. The polarised intensity is defined as $P_{\mathrm{I}}=\sqrt{Q^{2}+U^{2}}$, the degree of polarization $P=P_{\mathrm{I}} / I$, where $I$ is the total intensity and the polarization angle, $P_{\Theta}=0.5 \arctan \frac{U}{Q}$ defines the orientation of the polarization plane. The data analysis is described in more detail by Canovas et al. (2011). The design of ExPo includes a polarisation compensator which reduces the instrumental polarisation of $\sim 3 \%$ to the order of $10^{-3}$. This is removed in the data analysis by assuming that the central star is unpolarised.

The image of $\mathrm{R} \mathrm{CrB}$ in linear polarisation is shown in Fig. 1. To our surprise the image shows one clearly defined dust cloud, with a detection of $15 \sigma$, to the southeast (left) of the star which is indicated by a speckled blob. The cloud to the left is the scattering cloud, Cloud S, which shows clearly defined polarisation vectors. The systematic/noise error on the orientation of these vectors is $10 \pm 2^{\circ}$. The black contour line at the centre of the image indicates the FWHM of the stellar PSF.

\section{HST observations}

HST images of R CrB were retrieved from the MAST data archive (Clayton et al. 2011). These images were secured on 14 April 2009 using HST/WFPC2 (Wide Field Planetary Camera) imaging in broad-band $V(F 555 W)$ and $I(F 814 W)$ filters. These observations are summarised in Table 1 . The individual exposures taken with each filter were aligned and combined 
S. V. Jeffers et al.: Direct imaging of a massive dust cloud around R Coronae Borealis

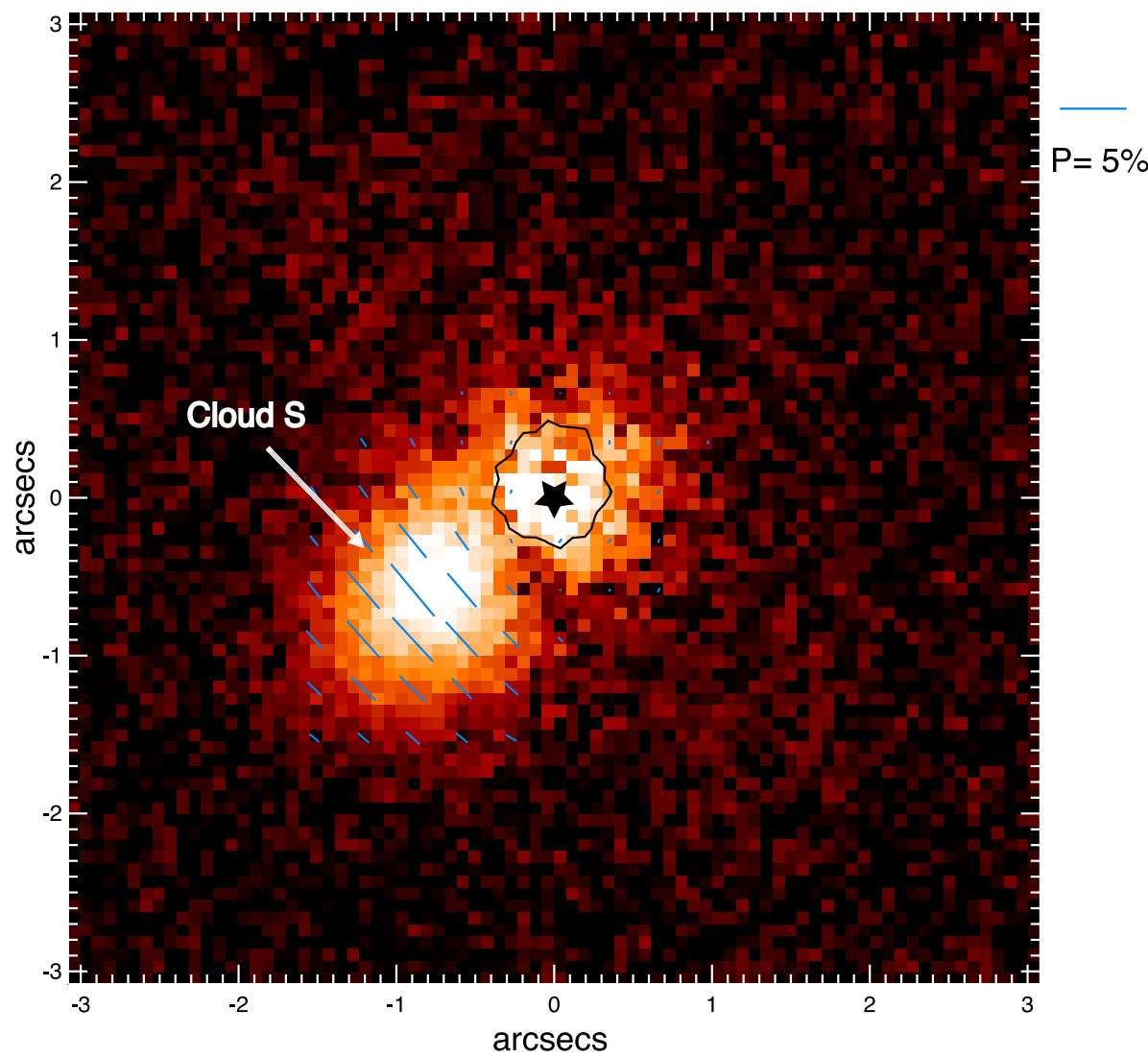

Fig. 1. ExPo image of the circumstellar environment of R CrB in linear polarisation. This image was taken at visible wavelengths (500-900 nm). The scattering dust cloud (Cloud S) is located to the south-east (left) of the image at 1.3" from the star. North is upwards and the scale of the image is in arcseconds.

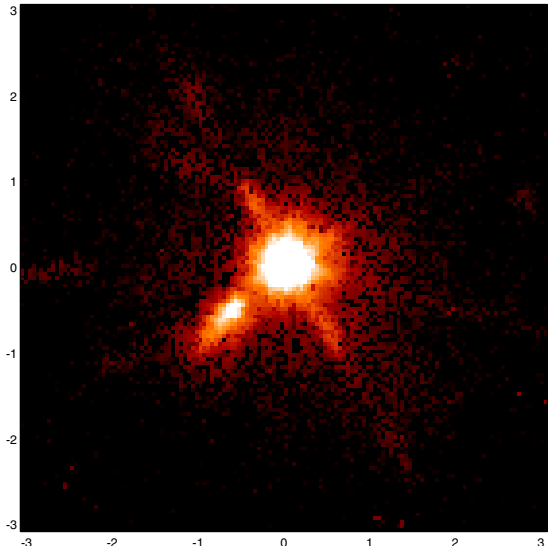

Fig. 2. HST/WFPC2 image of the circumstellar environment of $\mathrm{R} \mathrm{CrB}$. This image was taken with the $F 555 \mathrm{~W}$ filter (left). The scale of the image is approximately $6 \times 6$ arcsec.

with the STSDAS task gcombine with a cosmic ray rejection algorithm. There were 5 images for the $V$ filter and 4 for the $I$ filter, respectively giving a total exposure time of $487 \mathrm{~s}$ and $496 \mathrm{~s}$. Only the planetary camera (PC) part of the images, where R CrB is centred, are used. The spatial scale for the PC is $0.046^{\prime \prime}$ pixel $^{-1}$ and its field of view is $37^{\prime \prime} \times 37^{\prime \prime}$. The HST observations from April 2009 show an extended dust cloud (Cloud S) at the same location as shown in the ExPo images. The processed images for filters are shown in Fig. 2, magnified to match the field-of-view of ExPo.
Table 2. Magnitude of $\mathrm{R} \mathrm{CrB}$ at each epoch of observation and also during its bright unobscured state.

\begin{tabular}{lccc}
\hline \hline Filter & 14 April 2009 & 27 May 2010 & Unobscured \\
\hline$V$ & 15.00 & 14.45 & 5.91 \\
$B$ & 15.65 & 15.15 & 6.49 \\
$I$ & 14.10 & 13.55 & 5.31 \\
\hline
\end{tabular}

Notes. Data is from the AAVSO (American Association of Variable Star Observers).

\section{Observational facts}

The magnitude of $\mathrm{R} \mathrm{CrB}$ is shown in Table 2 at the epoch of the ExPo and HST observations. At the time of observation $\mathrm{R} \mathrm{CrB}$ is clearly in a minimum state with $M_{V}=15.0$, which started in July 2007. The overall reduction in flux, from $M_{V}=5.91$ to 15.00 , is approximately 4000 . If the obscuring cloud acts like a natural coronograph completely obscuring the star, then the only contribution is from light scattered from the dust in the surrounding halo. To obtain a magnitude drop of $\approx 9$ mag the scattered flux must be less than $1 / 4000$ of the stellar flux.

\subsection{Photometric variations}

The photometric light curve of $\mathrm{R} \mathrm{CrB}$ has been monitored for more than 150 years. During obscuration its light curve is characterised by a sharp decline followed by a gradual return to maximum light levels. There are also significant colour changes during obscuration. Plots of $V$ versus $B-V$ typically show (e.g. Fig. 5 from Efimov 1988) a very steep decline at the onset of 


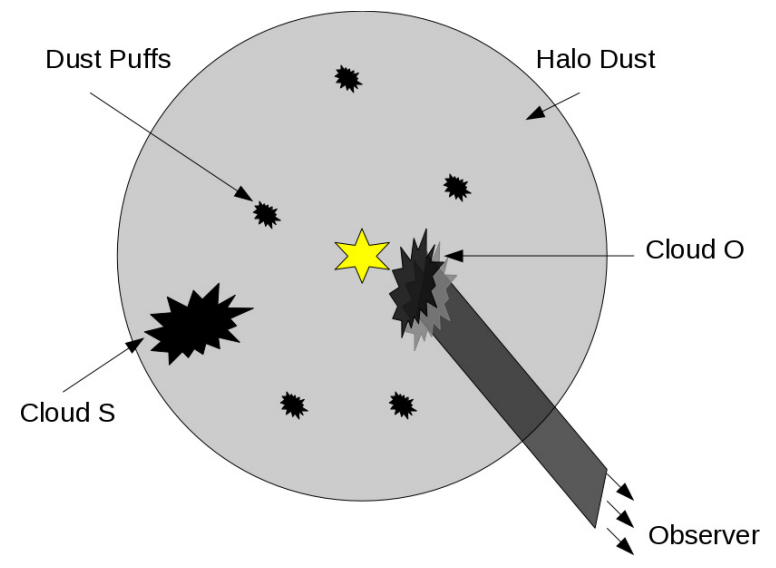

Fig. 3. Cartoon drawing showing the locations of the three distinct populations of dust around $\mathrm{R} \mathrm{CrB}$.

obscuration followed by reddening and then a shift towards very blue $B-V$ values just before the lowest light level. On the return to maximum light levels the light curve first reddens and then becomes bluer before reaching its normal light level. Colour declines that vary from the standard model will be discussed later in Sect. 5.

\subsection{Observed dust cloud properties}

The ExPo and HST observations, in scattered light, show that there are two dust clouds around $\mathrm{R} \mathrm{CrB}$. The first, Cloud $\mathrm{O}$, is the obscuring cloud which is responsible for the decrease in $\mathrm{R}$ CrB's brightness. The second cloud, Cloud S, the scattering cloud, is detected by HST and in linearly polarised light by ExPo. Additionally, in this analysis we consider that there is a third location of dust in the halo surrounding the star and is referred to in this work as Halo Dust. The locations of these dust populations are shown schematically in Fig. 3.

\subsubsection{Cloud S}

The properties of Cloud $\mathrm{S}$ are determined via aperture photometry (with a radius $=0.47^{\prime \prime}$ ) of the ratio of the flux in Cloud $\mathrm{S}$ to the total flux of the star. From the ExPo observations, the ratio (total stellar intensity)/(polarized cloud $S$ intensity) $=235$. Similarly for the HST images, the $F 555 W$ filter gives a Star/Cloud S ratio of 42 and with the $814 \mathrm{~nm}$ filter a ratio of 56 .

\subsubsection{Halo dust}

The Halo dust is defined as the halo of dust surrounding R CrB which is confirmed observationally by $L$-band observations staying constant during obscuration. Previous measurements of the infrared IRAS fluxes of R CrB (Lambert et al. 2001) show that $30 \%$ of the stellar flux is reprocessed by absorption of stellar light by the halo grains and subsequent reemission at infrared wavelengths. In the optically thin approximation the total amount of reprocessed light in the infrared is directly proportional to the absorption optical depth through the equation:

$f_{\mathrm{IR}}=1-\mathrm{e}^{-\tau_{\mathrm{abs}}}$,

where $\tau_{\mathrm{abs}}$ is the mean optical depth for absorption averaged over the stellar spectrum, and $f_{\mathrm{IR}}$ is the fraction of the light reprocessed in the infrared. For R CrB, $f_{\mathrm{IR}}=0.3$ and consequently $\tau_{\mathrm{abs}}=0.36$.
The fraction of light that is reprocessed by scattering, $f_{\text {scatt }}$, must be extremely small as the integrated intensity of the system, which is the sum of the starlight and the scattered light, can drop $9.1 \mathrm{mag}$ in the visual when the star is obscured. The fraction of scattered light can be equated as follows:

$f_{\text {scatt }}=1-\mathrm{e}^{-\tau_{\text {scatt }}}$,

where $\tau_{\text {scatt }}$ is the scattering optical depth. The observed decrease is $f_{\text {scatt }}<1 / 4000$ (9 mag), which means that $\tau_{\text {scatt }}<2.5 \times 10^{-4}$. The single scattering albedo of the grains must be

$\omega=\frac{\tau_{\text {scatt }}}{\tau_{\text {scatt }}+\tau_{\text {abs }}}<7 \times 10^{-4}$.

Consequently, the albedo of the grains in the visual is extremely small, i.e. on the order of $0.07 \%$.

\subsubsection{Cloud O}

The properties of Cloud $\mathrm{O}$ are derived from the wavelengthdependent extinction as the brightness of $\mathrm{R} \mathrm{CrB}$ decreases. During R CrB's bright state the $V$-band magnitude is 5.91 and its $B$ band magnitude is 6.49 , while during obscuration, the $V$-band and $B$-band magnitudes are 14.45 and 15.15 respectively (on 27 May 2010). These values are summarised in Table 2.

\section{Models of dust clouds}

In this section we use the observed properties of the dust clouds to deduce the properties of the dust grains in Cloud O, Cloud S and in the Halo Dust surrounding R CrB. The dust grains are assumed to be composed of amorphous carbon using the refractive index from Preibisch et al. (1993) and the particle shape model from Min et al. (2005).

Cosmic dust grains are not perfect homogeneous spheres. When dust grains are modelled using Mie theory, i.e. assuming the grains are perfect homogeneous spheres, the scattering phase function and degree of polarization show sharp resonances at certain scattering angles. Furthermore, the overall behavior is very different from that of more irregularly shaped grains, and even the sign of the polarization is often wrong (see e.g. Muñoz et al. 2004, Fig. 10). Exact computations of the scattering properties of realistically shaped grains are computationally demanding and beyond the scope of this paper. Fortunately, breaking the perfection of a homogeneous sphere in even the most simple way, suppresses the resonance effects significantly, for e.g. using the model by Min et al. (2005). For the computation of the optical properties we use this model for the grain shape with $f_{\max }=0.8$ representing rather irregularly shaped grains. This parameter is varied to estimate the error on the derived parameters. Throughout the analysis we fix the shape and composition of the dust grains and vary only their sizes. Since we consider irregularly shaped grains we refer to the volume equivalent radius.

\subsection{Halo dust}

To determine the grain properties of Cloud $\mathrm{O}$, it is first necessary to disentangle the contribution from the surrounding Halo dust to the photometric light curve. As explained in Sect. 4.2.2, the albedo of a dust grain is very sensitive to the size of the dust grain with small grains also having a low albedo. Consequently, it is necessary to make the grains very small, i.e. smaller than $5 \mathrm{~nm}$ to obtain an albedo of $0.07 \%$. This therefore implies that the grains in the surrounding halo are much smaller than those in Cloud S. 
S. V. Jeffers et al.: Direct imaging of a massive dust cloud around R Coronae Borealis

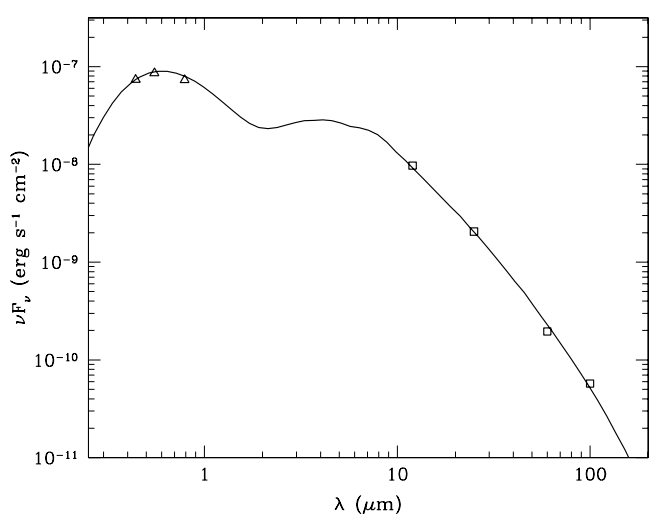

Fig. 4. The modelled fit to the SED of R CrB using the DHS grain model of Min et al. (2005) and the radiative transfer code MCMAX of Min et al. (2009). The optical data points are taken from Table 2.

\subsubsection{Fitting the SED}

As previously discussed, to match a dust reprocessing of $30 \%$ and decrease of 4000 at visible wavelengths it is necessary to assume that these particles do not scatter very efficiently and consequently are very small. To model the SED of R CrB these grains are set to be $5 \mathrm{~nm}$ in radius since R CrB's infrared excess is defined by its Halo dust. For the star we assume a blackbody with $T_{\text {eff }}=6750 \mathrm{~K}$ and $R_{*}=73.4 R_{\odot}$, at a distance of 1400 parsec (from Yudin et al. 2002; Asplund et al. 1997). A homogeneous low density dusty wind is included in the models as a static outflow, with a velocity of $200 \mathrm{~km} \mathrm{~s}^{-1}$ (Clayton et al. 2003). The IRAS fluxes from Walker (1985) are fitted by varying the mass loss rate. A dust mass loss rate of $7.5 \times 10^{-9} M_{\odot} \mathrm{yr}^{-1}$ is computed to provide the best fit as shown in Fig. 4. This result is similar to that obtained by Yudin et al. (2002) though they use a much slower wind $\left(45 \mathrm{~km} \mathrm{~s}^{-1}\right)$ and consequently determine a lower dust mass loss rate $\left(3.1 \times 10^{-9} M_{\odot} \mathrm{yr}^{-1}\right)$. The best fit model reproduces the global properties of the ISO spectrum of Walker et al. (1999).

\subsection{Obscuring cloud}

A characteristic component of the photometric light curve of $\mathrm{R} \mathrm{CrB}$ is that there are significant colour changes during obscuration, which can be used to constrain the grain properties of the obscuring dust cloud.

\subsubsection{Characteristic light curve properties}

$\mathrm{R}$ CrB's light curve typically shows first a sharp decrease in its $V$ magnitude while $B-V$ remains constant. This leads to a reddening of the star as the brightness declines further, followed by the star becoming bluer until it reaches its minimum. On the return to normal brightness levels the light curve first reddens before becoming bluer (see e.g. Efimov 1988, Fig. 5 for $1983 / 4$ minimum). The variations in colour are found to vary with the depth of the minimum, with shallower minima showing the characteristic drop in $V$, while $B-V$ remains constant, and subsequent red colour variations but are not followed by the starlight becoming bluer before minimum. An example of this is in the shallow minimum of January 1999, where $M_{V}$ decreases to only 9.5. For deep obscurations the general behaviour begins with a sharp decrease in $V$ while $B-V$ remains constant. It is only just before the minimum light level that the light curve becomes bluer before returning to maximum brightness levels following the standard behaviour i.e. first becoming more red and then more blue.

\subsubsection{Models of colour variations}

To understand how the sizes of the dust grains in an obscuring "dust puff" cause the observed colour changes, we have modelled an obscuring dust cloud from formation to dissipation. The characteristic sharp drop in $V$ magnitude, while $B-V$ remains constant, indicates the formation of large grey grains while the dust cloud is still thick and dense. This means that the grain sizes are at least $0.2 \mu \mathrm{m}$, which is the smallest possible size that can give grey extinction. As the cloud expands, and consequently has a lower density, only small particles (i.e. $5 \mathrm{~nm}$ ) can form, resulting in the onset of the reddening in the $V$ versus $B-V$ plot. When the star reaches minimum light, the cloud is then assumed to dissipate before $\mathrm{R}$ CrB returns to maximum light levels. The slope of the curve on return to maximum brightness is determined by the size distribution of the grains.

The obscuring dust cloud is modelled with a total optical depth equal to 1,3 and 10 which is only caused by the large grains. The small grains are added with an optical depth ratio of 3 small grains for every large grain. This small:large ratio of 3:1 was chosen to roughly reproduce the light curve by Efimov (1988). Consequently the total optical depths at minimum light are $\tau=12,4$ and 40 the results of which are respectively shown in Fig. 5 top, middle and lower panels. For the model that uses $\tau=4$, the minimum brightness level only reaches $M_{V}=9.9$ and is comparable to the obscuration in January 1999 where $M_{V}$ only decreased to 9.5. As with all models, large particles are formed at the beginning, with a sharp vertical decrease in $V$ versus $B-V$, and then small particles are formed leading to a reddening of the curve. In this case for low optical depth, there is no impact from the surrounding Halo dust cloud. For $\tau=12$, the curve begins with the same characteristic behaviour but shows an onset of reddening by small grains beginning at $\approx M_{V}=9$, followed by a bluing due to scattering from the Halo dust at $\approx M_{V}=12.5$. The $V$ magnitude then reaches a minimum of $M_{V}=14$ before reddening significantly on its return to maximum brightness levels. For the model with the highest optical depth i.e. $\tau=40$, the minimum light can be reached by using only very large grains (Fig. 5 lowest panel). As with the other models discussed here, the return to maximum brightness is characterised by a reddening. This is caused by the dispersal of the dust cloud, which at this time would also contains a significant fraction of very small grains.

\subsubsection{Comparison to observed colour variations}

The $V$ versus $B-V$ light curve of the current decline, is shown in Fig. 6. At minimum $V$ magnitude there are many colour variations of up to $B-V=0.5$ which is an intrinsic property of $\mathrm{R} \mathrm{CrB}$ stars (see Sect. 1). Although the light curve is missing several data points at the start of the obscuration, the models of the colour variations are in general agreement with the observed colour variations. The best fitting model is for $\tau=40$ which is also plotted in Fig. 6. To investigate the impact of multiple scattering we computed a full radiative transfer model for several points on the $V$ versus $B-V$ plot using MCMAX (Min et al. 2009). The results show that the inclusion of multiple scattering has the effect of making the initial sharp drop in $V$ bluer. To fit the observations, it is necessary to compensate for this by assuming a smaller grain size of approximately $0.13 \mu \mathrm{m}$. 

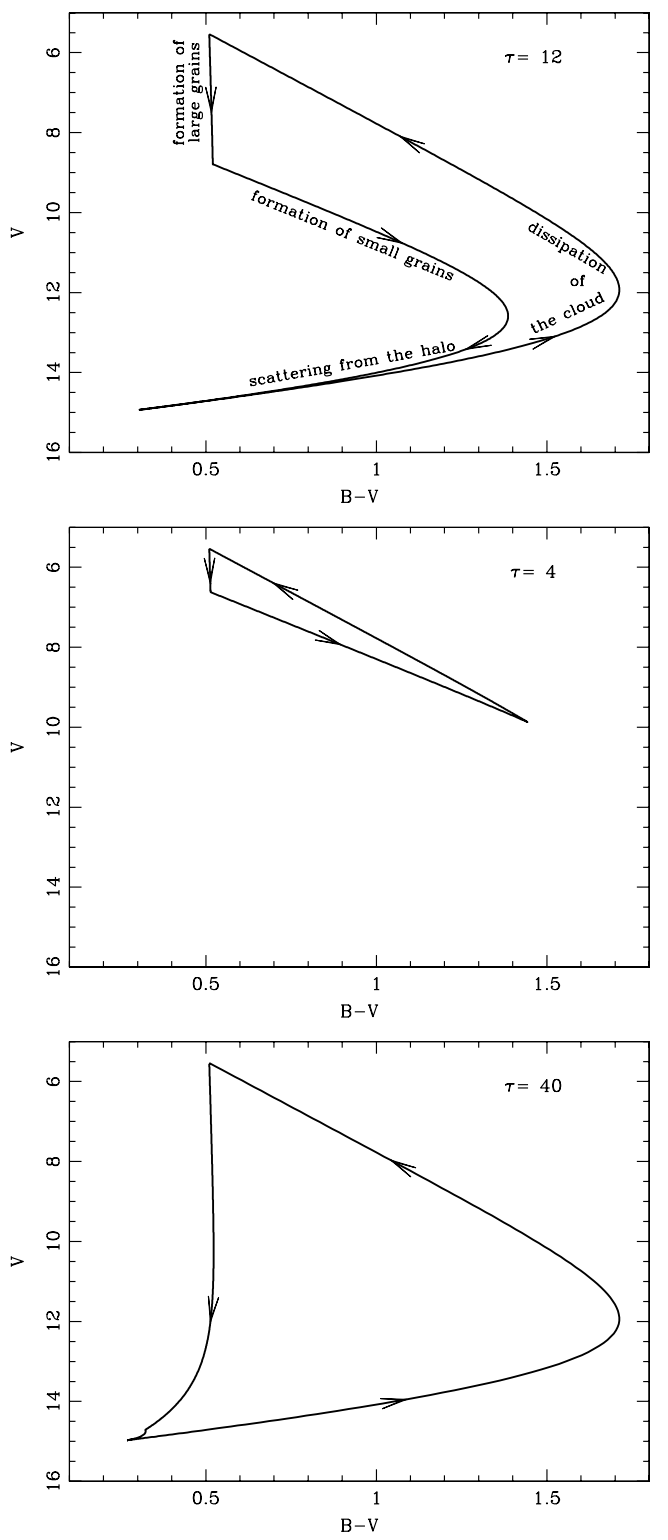

Fig. 5. Models of how the optical depth of the obscuring cloud impacts the resulting photometric light curve variation during obscuration, see Sect. 5.2.2 for explanation. For comparison models are shown for $\tau=12$ (normal obscuration), 4 (light obscuration), and 40 (dense obscuration) respectively for top, middle and bottom plots.

\subsection{Scattering cloud}

Observations in scattered light of Cloud $\mathrm{S}$ were taken by both HST and ExPo. In general, the colour of scattering is highly dependent on the scattering angle, as is the degree of polarization. The light scattering properties of the dust in Cloud $\mathrm{S}$ is determined using the models of Min et al. (2005). To fit the observations it is necessary to model a grain size and a scattering angle that simultaneously match the the polarized intensity of the ExPo images and the colour of the HST images.

The degree of polarisation is derived to be $>15 \%$ from the estimate of the polarized intensity of the ExPo image. As the ExPo image is not photometrically calibrated, we estimate the polarized intensity from the ratio between the total intensity of the star from the HST image and the polarized intensity from the cloud. In order to derive the properties of the cloud we computed the scattering properties of particles with sizes ranging from $5 \mathrm{~nm}$ up

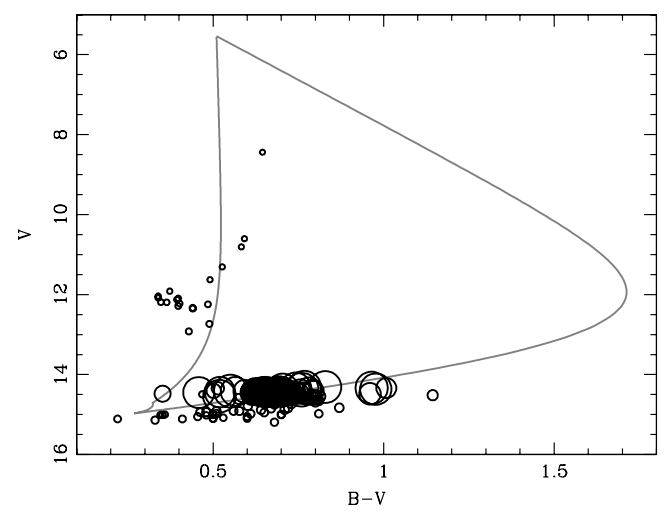

Fig. 6. The variation of $B-V$ with decreasing $V$ magnitude of the current obscuration, with the $\tau=40$ model overplotted. The size of the plotted circle increases with time. Note that the first points are not evenly spaced in time for the current obscuration. At minimum $V$ magnitude there are many colour variations of up to $B-V=0.5$ which is an intrinsic property of $\mathrm{R} \mathrm{CrB}$ stars. The photometric data are from the AAVSO.

to $0.2 \mu \mathrm{m}$ with irregularity parameters $f_{\max }=0.2-0.8$. The irregularity parameter was varied to determine how it impacts the derived particle size. Assuming a single size for the dust grains we infer that the grains are $\sim 0.14 \pm 0.02 \mu \mathrm{m}$ in diameter. The error on this value is derived from varying the irregularity parameter and shows that it has little impact on the derived particle size. A significantly smaller grain size results in a colour for Cloud $\mathrm{S}$ that is much bluer than the HST images, while using a larger grain size produces a result that is too grey. The scattering angle that matches all of the observations is about $\theta=94 \pm 33^{\circ}$ (i.e. Cloud $\mathrm{S}$ is located approximately in the observation plane of the star). From our models we can also derive the mass of the cloud which depends on the scattering efficiency (i.e. particle grain size and the scattering angle). We derive a dust mass of the cloud of $3_{-1}^{+4} \times 10^{-4} M_{\oplus}$ and an intrinsic degree of polarization of the cloud of $\sim 20 \pm 5 \%$.

\subsection{Summary of models}

It is evident from the SED that there must be a significant dust mass surrounding $\mathrm{R} \mathrm{CrB}$ since roughly $30 \%$ of the light is reprocessed as thermal emission. The very low scattering efficiency of these grains, as inferred from R CrB's minimum brightness, requires that these grains are very small. Contrary to this we find, from the scattered light by the dust cloud seen in the HST and ExPo images, that the grains in the dust clouds must be much larger with a size of approximately $\sim 0.14 \pm 0.02 \mu \mathrm{m}$. Also, the grey onset of the obscuration is evidence for the formation of very large grains when the dust clouds are dense.

\section{Discussion}

\subsection{Grain sizes}

\subsubsection{Halo dust}

The $V$-band magnitude of $\mathrm{R} \mathrm{CrB}$ was 14.45 and 15 respectively at the epochs of the ExPo and HST observations. Since R CrB is obscured by a dust cloud, this magnitude is assumed to be the brightness of the surrounding Halo dust. To simultaneously fit the visible magnitude decrease and the SED, it was therefore necessary to use $5 \mathrm{~nm}$ grain sizes since their albedo is very low and are hence very inefficient at scattering light. A larger grain size would have a larger albedo and it would not be possible 
to obtain the observed decrease in visual brightness. The $5 \mathrm{~nm}$ grain size is at the lower end of the MRN, Mathis, Rumpl, \& Nordsieck (Mathis et al. 1977), size distribution. An extremely small particle size for the Halo dust of R CrB has also been found by Yudin et al. (2002). This result is consistent with the observations of V 854 Cen of Whitney et al. (1992) where they state that the scattered flux may arise in the same clouds contributing to the observed IR flux if the albedo is low.

However, Ohnaka et al. (2001), from interferometric observations, show that the visibility function and spectral energy distribution can be simultaneously fitted with a model of an optically thin dust shell at maximum light but not at minimum light. They suppose that the discrepancy is attributed to thermal emission from a newly formed dust cloud, but the results from this paper and that of Yudin et al. (2002) conclude that the discrepancy of Ohnaka et al. (2001) can be resolved by assuming very small particle sizes, which are very inefficient at scattering starlight.

\subsubsection{Obscuring cloud}

Currently Cloud O totally obscures the star and we only see the scattered light from the low density halo making it impossible to probe its full size distribution. As previously described, the best fitting model to the observed colour changes is for $\tau=40$, where total obscuration can be reached with only large grains, and is overplotted in Fig. 6.

\subsubsection{Scattering cloud}

Both the ExPo and HST images of the circumstellar environment of R CrB clearly show the presence of a large and extended dust cloud, Cloud S. The cloud is surprisingly elongated and could indicate how dust clouds interact with their surroundings. The comprising dust is inferred to be $0.14 \pm 0.2 \mu \mathrm{m}$ in diameter from combining the light scattering properties of the dust (Min et al. 2005) with the observed colour and degree of polarisation. The derived sizes of grains in Cloud $\mathrm{O}$ and Cloud $\mathrm{S}$ are in good agreement. Cloud $\mathrm{S}$ is significantly older than the recently ejected Cloud $\mathrm{O}$, having been ejected at least 50 years ago, assuming a constant outflow speed of $200 \mathrm{~km} \mathrm{~s}^{-1}$ (see Sect. 6.3). This indicates that there is no significant grain evolution due to, for example, the high velocity wind that contains many small particles. Additionally from the combination of the ExPo image with the two HST images, we infer that the scattering angle of the dust is approximately $\theta=94 \pm 33^{\circ}$ i.e. almost perpendicular to the observer.

From our results we derive Halo Dust grain sizes of $5 \mathrm{~nm}$, Cloud $\mathrm{S}$ grain sizes of $0.14 \pm 0.2 \mu \mathrm{m}$ and Cloud $\mathrm{O}$ grain sizes of $0.13 \mu \mathrm{m}$. To investigate the impact of using slightly different grain sizes, we added an size distribution of particles, modelled by delta functions at $5 \mathrm{~nm}$ and $0.2 \mu \mathrm{m}$. This has the effect of reducing the dust mass in Cloud S. However, it is still necessary to include a large number of these very small grains. The derived mass of Cloud S clearly depends on the size of the composing dust grains. For the case where the grain size $=0.14 \pm 0.2 \mu \mathrm{m}$, the dust cloud mass is $3_{-1}^{+4} \times 10^{-4} M_{\oplus}$. This is a minimum mass as these grains are the most efficient at scattering while still fitting the colour information from the ExPo and HST images. The mass of Cloud $\mathrm{S}$ when composed of grains that follow the size distribution, can only be greater than the mass derived for $0.14 \mu \mathrm{m}$ grains because this distribution contains many more grains that scatter less efficiently. However, the increased mass of the model using the size distribution is perhaps more realistic.

\subsection{Why are there different grain sizes?}

The different grain sizes in Cloud $\mathrm{S}$ and $\mathrm{O}$ and in the Halo dust could be explained by models of the formation of dust-driven winds around late type carbon stars (Gail \& Sedlmayr 1987) According to this model, the dust is dominated by very large grains close to the star, but further out the atoms can only form very small nuclei, due to the decreased density. For the case of $\mathrm{R} \mathrm{CrB}$, large grains could be formed for as long as the dust cloud stays sufficiently dense. If a significant fraction of the carbon remains after the cloud begins to disperse, then the remaining carbon will be in a low density environment and consequently will only be able to form very small nuclei. This is also what happens in the low density halo, where only very small grains can form.

\subsection{Stellar wind}

The stellar wind of R CrB has been measured by Clayton et al. (2003) to be $200 \mathrm{~km} \mathrm{~s}^{-1}$ from an analysis of the He I $\lambda 10830$ line. Notably, R CrB shows a P Cygni or asymmetric blue-shifted profile at all times, i.e. during both minimum and maximum light indicating that the stellar wind is independent of the ejection of "dust puffs". This is consistent with the model presented in this paper. The nature of the stellar wind is highly likely to be dust driven. According to the theoretical models of Gail \& Sedlmayr (1986), a dust driven stellar wind is possible in the case of cool Carbon stars with a mass loss rate of the order of $10^{-6} M_{\odot} \mathrm{yr}^{-1}$ and with a non-negligible dust production rate. The computed dust mass loss rate for $\mathrm{R} \mathrm{CrB}$ is $0.9 \times 10^{-6} M_{\odot} \mathrm{yr}^{-1}$ (from Sect. 5.2.1), though the conditions for a dust driven wind on $\mathrm{R} \mathrm{CrB}$ are enhanced since it is hydrogen deficient star and because there is enormous radiation pressure on the dust grains.

Polarimetric observations could indicate the presence of permanent clumpy non-spherical dust shells (Clayton et al. 1997; Yudin et al. 2002). Indeed if the "dust puffs" are ejected from $\mathrm{R} \mathrm{CrB}$ at a frequency equal to $\mathrm{R}$ CrB's 50 day pulsation period (Crause et al. 2007), at a distance of $2 R_{*}$, there should be a very large number of them between the edge of the Halo dust and the star. This frequent ejection of dust puffs in combination with the dusty stellar wind are considered to be the dust feeding mechanisms for the circumstellar Halo dust.

\subsection{Dust clouds}

The observations in this paper confirm the "dust puff ejection" model of R CrB first proposed by Loreta (1934) and O'Keefe (1939). The HST and ExPo images clearly show a dust cloud at a detection signal-to-noise of 33 sigma. Observations of another R CrB star RY Sgr (de Laverny \& Mékarnia 2004; Bright et al. 2011) show many dust clouds likely to be randomly ejected from the stellar surface. As noted by de Laverny \& Mékarnia (2004) over the last 50 years the number of brightness declines for $\mathrm{R} \mathrm{CrB}$ is much greater than that of RY Sgr, implying that we should be seeing many more dust clouds in the circumstellar environment of R CrB.

Tests in the laboratory have shown that ExPo can reach contrast ratios of up to $10^{-5}$ which is much fainter than the Cloud S:star ratio of $10^{-2}$. To determine if there are fainter structures also present in the ExPo images we have smoothed the data using a Gaussian kernel filter, with a width of $1^{\prime \prime}$, to reduce the 


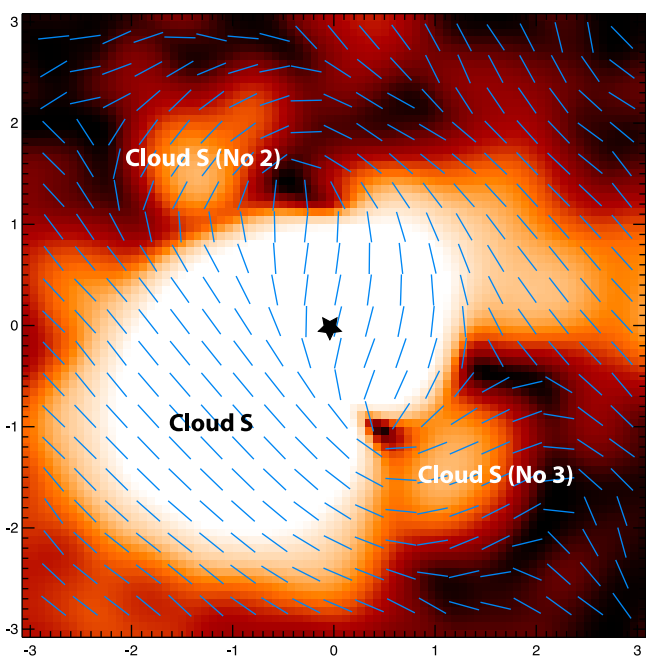

Fig. 7. Smoothed ExPo image to decrease noise effects, showing a tentative detection of additional dust clouds present at $10^{-5}$ level.

contribution of noise. The resulting image is shown in Fig. 7, where the shown vectors are scaled to the degree of polarisation. In addition to the Cloud $S$, clearly seen in the ExPo images shown in Fig. 1, there is a tentative detection of two coherent structures which could be two additional dust clouds. These clouds are located just left of centre at the top of the image and just right of centre at the bottom of the image. The tentative detection is plausible because the dust cloud/star contrast ratio is $10^{-5}$, they have been detected in all of the ExPo observations and the polarisation vectors are correctly aligned. They are unlikely to be due to spurious instrumental artifacts and do not appear in ExPo images of stars without circumstellar matter. Future instrumentation such as SPHERE at the VLT and HiCIAO on Subaru will be able to confirm this result.

By assuming a "dust puff" formation radius of $2 R_{*}$ and a velocity of $200 \mathrm{~km} \mathrm{~s}^{-1}$, we derive that Cloud $\mathrm{S}$ was ejected 50 years ago. A surprising factor is that it still remains intact and easily detectable meaning that it must have been related to an exceptionally large ejection of dust. If the cloud has a higher density, due to the abnormal mass of the cloud, it could form a much bigger fraction of large, and consequently high-albedo, grains. If the same age calculation is applied to the current obscuring cloud, Cloud $\mathrm{O}$, and assuming that it will continue to travel along the line of sight, it could imply that $\mathrm{R} \mathrm{CrB}$ will remain obscured for many decades.

\section{Conclusions}

We conclude that there are two distinct grain populations in the circumstellar environment of $\mathrm{R} \mathrm{CrB}$. The first is a population of small $5 \mathrm{~nm}$ grains that comprise the low density stellar wind and the second population of large $(\sim 0.14 \mu \mathrm{m})$ grains that are formed in the ejected dust clouds. Our polarimetric images together with archival HST images, surprisingly reveal one exceptionally massive dust cloud composed of large grains. The current minimum of $\mathrm{R} \mathrm{CrB}$ is also noteworthy and lends additional support to the presence of large dust grains in ejected dust clouds.

Acknowledgements. We acknowledge support from NWO. We also thank the American Association of Variable Star Observers for the photometric data on $\mathrm{R} \mathrm{CrB}$ and the very helpful and knowledgeable staff at the William Herschel Telescope.

\section{References}

Asplund, M., Gustafsson, B., Kiselman, D., \& Eriksson, K. 1997, A\&A, 318, 521

Bright, S. N., Chesneau, O., Clayton, G. C., et al. 2011, MNRAS, 414, 1195 Canovas, H., Rodenhuis, M., Jeffers, S. V., Min, M., \& Keller, C. U. 2011, A\&A, 531, A102

Clayton, G. C. 1996, PASP, 108, 225

Clayton, G. C., Bjorkman, K. S., Nordsieck, K. H., Zellner, N. E. B., \& Schulte-Ladbeck, R. E. 1997, AJ, 476, 870

Clayton, G. C., Geballe, T. R., \& Bianchi, L. 2003, ApJ, 595, 412

Clayton, G. C., Sugerman, B. E. K., Stanford, S. A., et al. 2011, ApJ, 743, 44

Crause, L. A., Lawson, W. A., \& Henden, A. A. 2007, MNRAS, 375, 301

de Laverny, P., \& Mékarnia, D. 2004, A\&A, 428, L13

Donn, B. D. 1967, in Interstellar Grains, ed. J. M. Greenberg, \& T. P. Roark, NASA, Washington, SP-140, 191

Efimov, Y. S. 1988, Astronomicheskii Zhurnal, 65, 979

Fadeyev, I. A. 1988, MNRAS, 233, 65

Feast, M. W. 1979, in Changing Trends in Variable Star Research, ed. F. M. Bateson, J. Smak, \& I. H. Urch (Hamilton: Univ. Waikato Press), IAU Colloq., 46, 246

Feast, M. W. 1986, in Hydrogen Deficient Stars and Related Objects, ed. K. Hunger, D. Schoenberner, \& N. Kameswara Rao (Dordrecht: Reidel), IAU Colloq., 87, Astrophys. Space Sci. Lib., 128, 151

Gail, H., \& Sedlmayr, E. 1986, A\&A, 161, 201

Gail, H. P., \& Sedlmayr, E. 1987, A\&A, 171, 197

Hecht, J. H., Holm, A. V., Donn, B., \& Wu, C. 1984, ApJ, 280, 228

Kilkenny, D., \& Whittet, D. C. B. 1984, MNRAS, 208, 25

Lawson, W. A., Cottrell, P. L., Gilmore, A. C., \& Kilmartin, P. M. 1992, MNRAS, 256, 339

Leão, I. C., de Laverny, P., Chesneau, O., Mékarnia, D., \& de Medeiros, J. R. 2007, A\&A, 466, L1

Loreta, E. 1934, Astron. Nachr., 254

Mathis, J. S., Rumpl, W., \& Nordsieck, K. H. 1977, ApJ, 217, 425

Min, M., Hovenier, J. W., \& de Koter, A. 2005, A\&A, 432, 909

Min, M., Dullemond, C. P., Dominik, C., de Koter, A., \& Hovenier, J. W. 2009, A\&A, 497, 155

Muñoz, O., Volten, H., Hovenier, J. W., et al. 2004, J. Geophys. Res., 109, D16201

Ohnaka, K., Balega, Y., Blöcker, T., et al. 2001, A\&A, 380, 212

O'Keefe, J. A. 1939, AJ, 90, 294

Payne-Gaposchkin, C. 1963, ApJ, 138, 320

Preibisch, T., Ossenkopf, V., Yorke, H. W., \& Henning, T. 1993, A\&A, 279, 577

Pugach, A. F. 1977, Information Bulletin on Variable Stars, 1277, 1

Walker, H. J. 1985, A\&A, 152, 58

Walker, H. J. 1986, Irish Astron. J., 17, 318

Walker, H. J., Heinrichsen, I., Clayton, G. C., \& Rosenbush, A. E. 1999, in The Universe as Seen by ISO, ed. P. Cox, \& M. Kessler, ESA Noordwijk, ESA Spec. Publ., 427, 425

Whitney, B. A., Clayton, G. C., Schulte-Ladbeck, R. E., \& Meade, M. R. 1992, AJ, 103, 1652

Yudin, B. F., Fernie, J. D., Ikhsanov, N. R., Shenavrin, V. I., \& Weigelt, G. 2002, A\&A, 394, 617 